\title{
Design Scheme of Automatic Line Tracking Robot Based on MSC1210 MCU
}

\author{
Fu Zongjian \\ Department of Information Engineering \\ Zhengzhou Railway Vocational \& Technical College \\ Zhengzhou, China \\ zzrvtc123@126.com
}

\author{
Gao Hui \\ Department of Information Engineering \\ Zhengzhou Railway Vocational \& Technical College \\ Zhengzhou, China \\ zzrvtc123@126.com
}

\begin{abstract}
For the realization of the industrial production of automatic data collection and intelligent exploration missions, a line tracking robot based on MSC1210 MCU is designed. Through demonstration and comparison of several design schemes, completed the hardware design of automatic line tracking robot.The whole system of mechanical structure based on ordinary toy robot, can smoothly track pavement black orbit running, take full advantage of 24 bit high precision A/D converter of MSC1210 SCM, and can more conveniently complete the real-time and fast control of the robot movement .Experiments show that the design of hardware meet the requirements, the robot has the technical features of high intelligence, high performance and low cost. so it has a greater application value.
\end{abstract} PID

Keywords- Automatic tracking; Robot; MCU;MSC1210;

\section{INTRODUCTION}

Modern intelligent factories which are mainly characterized by informationization, automation, networking and intelligent are becoming technical characteristics of modern industrial. It is automatic tracking line robot that realizes the equipment of intelligent machining and automation production [1]. It is generally composed of automatic guiding system, power system, control and communication system and the security system. As a intelligent mobile simple robot [2], automatic tracking line robot is built in an organization and structure of complex engineering system based on computer, the autonomous function is implemented by the sensor and control information processing structure supported [2].

\section{THE OVERALL DESIGN SCHEME}

The block diagram of the overall design scheme of complete machine as Fig.1 Mainly includes the scheme design of robot body, the design of processor, the design scheme of SENSOR, the choice of obstacle detector, the choice of metal detector, the design of display circuit and so on.

\section{THE DESIGN SCHEME OF ROBOT BODY WALKING}

The body is the foundation of the whole system. Through repeated comparisons and demonstrations, developed the scheme of the left and right wheels driven separately, and the front with universal wheel steering. The left and right wheels respectively with two speed and torque is driven by basical same DC motor, the front is a universal wheel. So, when the two DC motors have same speed but in the opposite direction when the electric vehicle can achieve in-situ rotation, which can easily achieve the robot coordinate invariant $90^{\circ}$ and $180{ }^{\circ}$ turning. When the vehicle goes forward, the left and right wheels and a universal wheel make up three point structure. This structure makes the robot go forward smoothly, can avoid the situation that the front and rear wheels are too low so that the drive power of two driving wheel is not enough [3].

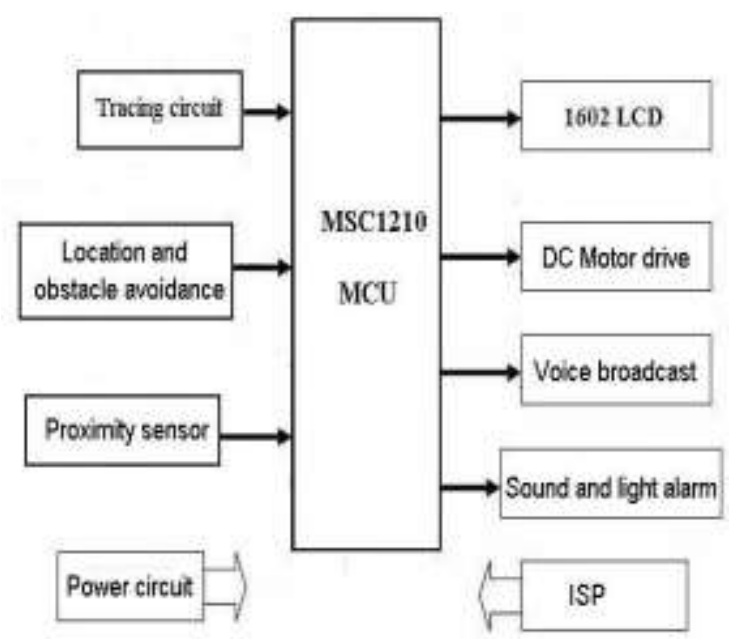

Figure 1. The overall design scheme of the robot

\section{THE CHOICE AND DESIGN OF PROCESSOR}

Scheme 1: Use programmable logic controller CPLD as processor. CPLD can realize the logic function of a variety of complex, large scale, high density, small size, high stability and rich IO resources [4], it is easy to extend its function.Use parallel input, output, and improves the processing speed of the system, suitable for the control core of large-scale control system. But the system does not require complex logic functions, the speed of data processing requirements are not very high, the hardware cost is relatively high.

Scheme 2: Using spCe061 MCU of Sunplus company, which is a 16 bit controller, and has the characteristics of small size, high driving capability, high integration, high reliability, low power consumption, simple structure and so on. High processing speed, especially in the field of speech signal processing and speech recognition. But 
when the Sunplus MCU application of speech processing and recognition, because of its occupied CPU resource more and makes Sunplus reduce the processing speed and capability of other tasks at the same time, the stability of the system is not too high.

Scheme 3: Use AT89S51 MCU of Atmel company as the processor. The AT89S51 is a low power, CMOS 8 bit MCU of 51 kernel of high performance, the chip containing the $8 \mathrm{~K}$ space can be repeatedly erased 1000 times Flash read-only memory, random data memory with 256 bytes (RAM), 32 IO ports and two 16 bit programmable timer counter. And the 51 single chip microcomputer in the series without programmer directly by the serial or parallel port can download the program to the MCU, disadvantage is poor encryption, no built-in A/D converter chip [5].

Scheme 4: Selection of TI MSC1210 MCU, all instruction functions of MSC1210 series are same as standard 8051, influence of the bit, mark and register are the same, only the timing is different, for technology developers are easy to use. MSC120 MCU used to streamline 8051 kernel, under the effect of the same external clock, its execution speed is 1.5 3 times faster than the standard 8051 (the difference between each instruction is 4 clock cycles and 12 clock cycles). In the same instruction and the clock, speed increased to more than 2.5 times.

Analog features of MSC1210 is: 24 Bits No Missing Codes. 22 Bits Effective Resolution at 10Hz--Low Noise: 75Nv. PGA From 1 to 128. Precision On-Chip Voltage Reference. 8 Differential/Single-Ended Channels. OnChip Offset/Gain Calibration. Offset Drift: $0.1 \mathrm{ppm} /{ }^{\circ} \mathrm{C}$. Gain Drift: $0.5 \mathrm{ppm} /{ }^{\circ} \mathrm{C}$. On-Chip Temperature Sensor. Burnout Sensor Detection. Single-Cycle Conversion. Selectable Buffer Input.

Digital features of MSC1210 is:8051-Compatible Package. High-Speed Core --4 Clocks per Instruction Cycle.DC to 33MHz.Single Instruction 121ns . Dual Data Pointer [6].

These features provide a good hardware foundation for the real-time tracking line robot [7]. Based on the above, select the scheme 4. MSC1210 pinout diagram as shown in Fig.2.

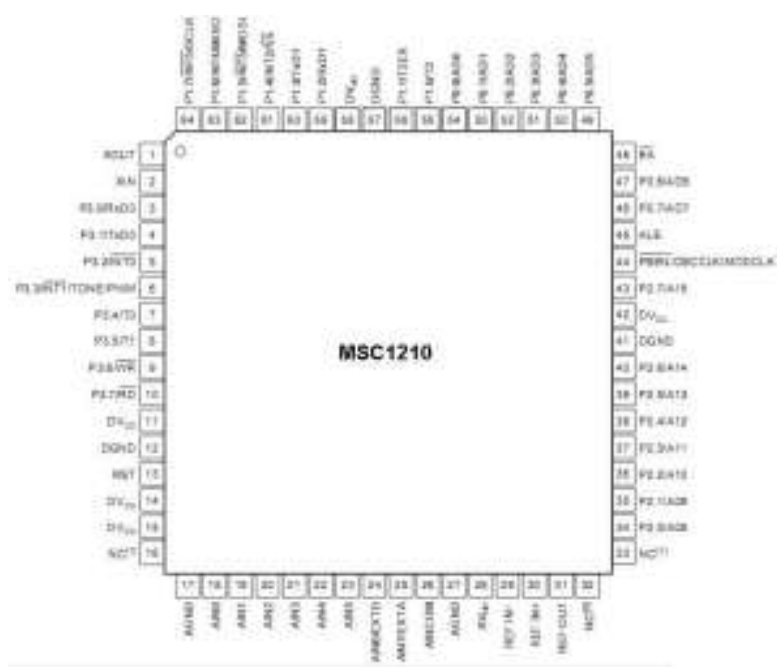

Figure 2. MSC1210 pinout diagram
V. THE SELECTION OF DESIGN SCHEMES OF OTHER HARDWARE CIRCUIT

\section{A. The selection scheme of tracing sensor}

Scheme 1: Photosensitive resistors tracing sensor.. The resistance of photosensitive resistor can follow the change of the ambient light and change. When light strikes the white above, light reflection is strong; light is exposed to the black line above, light reflection is weak. Therefore, the photosensitive resistance in white and black line above, resistance can happen obvious change. The resistance changing value after comparator can output high level. But this scheme by light effect is very big, can not work stably. Therefore consider other more stable solution.

Scheme 2: Using the infrared transmitting tube and the receiving tube fabricates photoelectric tracing sensor. The infrared emission tube emits infrared radiation, when the infrared radiation reflects after emitting to the white surface, if the infrared receiving tube can receive the reflected light, the result shows that it detects the white line and then output is low level, if not receiving transmitting tube light, the result shows that it detects Apodemus then output is high level. Tracing sensor that can meet the requirement of tracing, the launch tube is a gallium arsenide infrared emitting diode, and the receiving tube is a high sensitivity, silicon planar phototransistor. The infrared photoelectric tube tracing circuit has the advantages of simple structure, light effects of small, stable and practical. And through voltage comparator cooperation form tracing sensor circuit. Selection of scheme 2 .

The practical circuit scheme 2 as shown in Fig .3. The color signal for the robot recognition is mainly black, white two kinds of circumstances. Fig .3 depicts a group of 1 groups of infrared sensor circuit, the actual optimization design with the same 8 groups of track plate.

In Fig .3 the comparative voltage of voltage comparator is decided by a precision potentiometer RP1. D1 is the LED. When output voltage of the LM339 is low, LED will light, and so it is easy to debug tracing circuits.

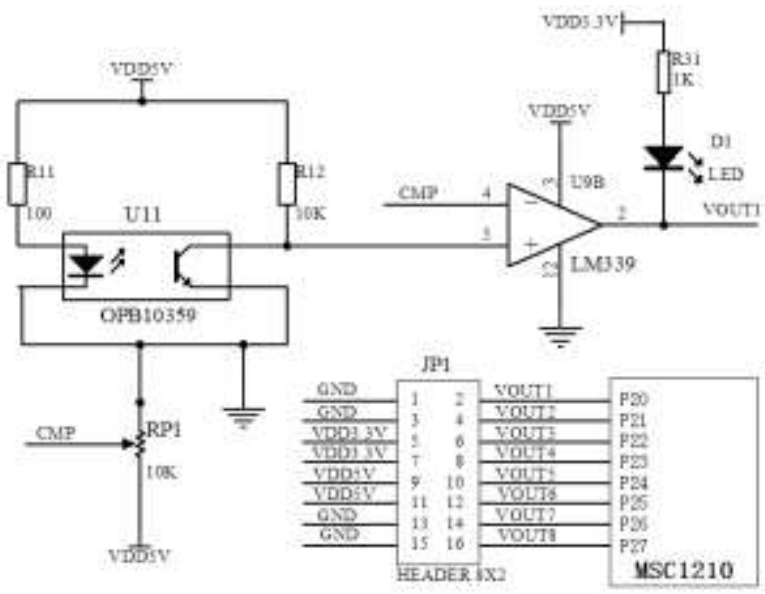

Figure 3. Tracing circuit

\section{B. The selection scheme of obstacle detector}

Scheme 1: Using infrared photoelectric switch for obstacle avoidance. The principle of photoelectric switch is based on a beam projector, is interdicted by an object 
or part of the reflection, the projector will judge and react, is the use of detected objects on the infrared beam shading or reflection, a synchronization loop gating and detection of objects have or no, the object is not limited to metal, and can detect for all the objects reflecting light. Photoelectric switch circuit lap is simple, easy to use. When the light is reflected back, output is low level. When there is no light is reflected back, output is high level. The low level can be just as MCU external interrupt. This scheme can detect simple obstacle, cannot meet the requirements of the system.

Scheme 2: Design of ultrasonic ranging and PSD ranging circuit, to realize vehicle location and intelligent obstacle avoidance function provides assurance. Ultrasonic sensors are for obstacle avoidance. The principle of ultrasonic sensor is: ultrasonic by piezoelectric ceramic ultrasonic sensor is issued, when the obstacle is reflected back, again received by ultrasonic sensor. Then the amplified signal is sent to the MCU. Ultrasonic sensors are widely used in the design of obstacle avoidance [8]. PSD (Position Sensitive Detector, position sensitive detector) is a photosensitive semiconductor device, with a structure similar to port diode. The receiving light beam from the outside, the output current of PSD sensor and the optical receiver point position hold function relationship. The biggest characteristic of PSD -- position sensing properties and triangulation method, made up a distance sensor [9]. The GP2Y0A21YK sensor, the built-in LED (infrared light emitting diode) and PSD detector (Position Sensitive Detector) module. It outputs the distance of placing objects in front of the distance sensor by changing the output DC voltage output. As can be seen, scheme 2 is more reliable, and select scheme 2 .

\section{The design scheme of metal detector}

Scheme 1: Using the theory of eddy current sensor homemade metal detector. This scheme is easy, but difficult to debug, the output signal is not reliable, the success rate is relatively low, it is difficult to accurately output sensing information. Therefore consider other solutions.

Scheme 2: By using the inductance is easy to purchase on the market of industrial on the proximity switch, the circuit uses the more commonly used LJ12A3-4-Z/BY proximity switch to complete the detection task of iron. Although the inductive proximity switch volume is large, but the design is acceptable, and the output signal is very reliable, good stability, small interference. Therefore, we select the scheme.

\section{The design scheme of display components}

Scheme 1: Using the LED digital tube display. Digital tube as display high speed, the use of simple, concise and display effect has been widely applied. Disadvantage is the digital tube can not display more rich in content.

Scheme 2: Using 12864 LCD display. LCD because of its clear display, display rich content, display large information. 12864 LCD module is large volume, low cost, programming more trouble.

Scheme 3: Using 1602 LCD display. 1602 LCD module can be smaller, can display 2 lines of 32 characters, display content can completely meet the tracking line robot of the functional requirements of the system, in addition, 1602 LCD module is cheap, costeffective, simple software programming. So considering the display component, we select scheme 3.

\section{E. The selection scheme of motor}

Scheme 1: The stepping motor as driving motor of the system. Because of the rotation angle can be accurate positioning, precise positioning can be achieved and the position of the vehicle forward. But the output torque of the stepping motor is low, and decreased with the increase of rotational speed, and torque at higher speeds will fall sharply. Do not apply to the robot and so on that have a system which requires a certain speed and torque. On the basis of comprehensive consideration, to give up the plan.

Scheme 2: Using DC motor. DC motor with large torque, small volume, light weight, simple assembly, easy to use. The original is powered by a high-speed motor, drives the gear (gear) gear set, and can produce high torque. But also it is more convenient for control circuit. DC motor can better meet the system design requirements, so choose scheme 2 .

\section{F. The design of motor drive circuit}

Scheme 1: A motor drive circuit is made up of using discrete components. The drive circuit is composed of discrete elements, and has the advantages of simple structure, low price, widely used in practical applications. But the circuit performance is not stable enough. So give up the plan.

Scheme 2: Using special chip L298N as the motor drive. L298N is a high voltage and high current full bridge driver chip, its high response frequency, and can be used for PWM motor control. A L298N can respectively control two DC motors, but also with a control enables the end. The chip is used as a motor drive, convenient operation, good stability, excellent performance.

On the basis of comprehensive consideration, we select the scheme 2 .

\section{G. The design of the voice prompt circuit}

Scheme 1: The use of Sunplus streamline the development board spce61 board, the board has the advantages of small volume, convenient use, with voice strong processing ability, also has the speech and the speech recognition function. Sunplus MCU is 16 bit microcontroller, with DSP function, there is a strong information processing capability, and maximum clock frequency is $49 \mathrm{MHz}$, with the advantages of higher speed operation. These are for speech processing and recognition for the good condition. But increases the complexity of the system, is not easy to use the scheme.

Scheme 2: Selecting DSP for speech recognition, DSP has a strong information processing capability, recognition memory recording and voice to voice, but considering the system cost and flexibility and convenience, so give up the plan.

Scheme 3: Choosing a special speech memory chip ISD2560, the MCU record playback address is for controlling. This method is simple and convenient, the operation mode can be segmented recording, playback. Can the voice storage total 60s. The disadvantage is that the accounting for many $\mathrm{I} / \mathrm{O}$ ports. The circuit $\mathrm{I} / \mathrm{O}$ ports 
are relatively abundant, by comparing, then select scheme 3.

\section{H. Power circuit design}

Because the system needs to use battery powered, consider the following several ways for power supply system:

Scheme 1: using $12 \mathrm{~V}$ battery as the power supply for a robot. Battery with voltage output characteristics of strong current driving capability and stability. But the volume of the battery is too large, the small electric vehicles is extremely inconvenient.

Scheme 2: uses six $1.2 \mathrm{~V}$ rechargeable lithium battery in series a total of $7.2 \mathrm{~V}$ to supply power to the DC motor, after voltage transformation of 7805 for the microcontroller, sensors and motor power supply. Proved by the experiment, when the battery to supply power to the DC motor, working voltage single-chip, sensor, unstable performance of mutual interference is more serious.

Scheme 3: a dual power supply, a motor drive circuit, the other path three terminal regulator $7805 \mathrm{MCU}$ and sensor supply voltage. The motor drive power and control system of power separation, in order to increase the stability of the system, after the experiment, the effect is good.

On the basis of comprehensive consideration, we select the scheme 3 .

\section{THE ALGORITHM AND PROGRAMMING CONTROL OF DC MOTORS}

In this system, using $\mathrm{C}$ programming language to realize the motor control. MSC1210 microcontroller output pulse width modulation (PWM) signal to change the motor speed. The two ends of the motor voltage and waveform control the duty ratio, so the speed of the motor and the duty cycle is proportional to the duty ratio. The duty ratio is greater and the motor turn faster. After the $100 \mathrm{~Hz}, 1 \mathrm{KHz}, 10 \mathrm{KHz}, \mathrm{PWM}$ output drive motor that: when the frequency is $100 \mathrm{~Hz}$, the motor running in clearance rotating state; when the frequency is $10 \mathrm{KHz}$, the motor runs unstable; when the frequency is $1 \mathrm{KHz}$, different duty ratio motor operation is very smooth, rotating speed, change quickly. MSC1210 produces modulation waveform of motor when the PWM frequency is $1 \mathrm{KHz}$ the as Fig. 4 .

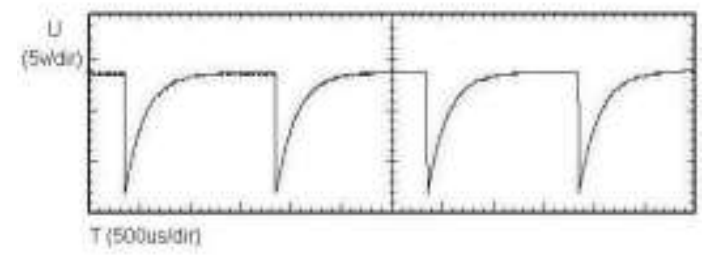

Figure .4 modulation waveform of the PWM frequency is $1 \mathrm{KHz}$

The method for control of DC motor,based on the collection of current feedback value and the motor speed, Using PID algorithm to control [10].

In PID control, proportional effect is to make quick response to the deviation, Kp bigger, stronger control ability, but excessive Kp would increase overshoot (more than a given value of volume). Integral action is deviation eliminating accumulated (i.e. static error), but integral can reduce the response speed, increasing the overshoot. Differential loop control according to the change trend of deviation, deviation change faster, differential link output is greater, and the deviation becomes larger before correction. $\mathrm{Kd}$ is greater, the greater the role of differential.

The speed of motor control using incremental PID control, AVR MCU procedures according to the feedback speed system left, right wheel motor current speed and set speed value, according to different conditions appropriate to change the $\mathrm{Kp}, \mathrm{Ki}, \mathrm{Kd}$ parameters, and then control the output 2 groups of PWM signal duty ratio, to achieve speed and direction change the role of the system, always in good working state, to achieve the purpose of accelerating, decelerating sensitive and good stability.

\section{CONCLUSION}

By the intelligent tracking robot test and practical running, the robot can find the way through the line specified before, stable operation, to arrive at the designated location, can perform distance measurement and real-time display different markers, and the liquid crystal display accurate data. The realization of intelligent robot each function validates the feasibility of the design of system software and hardware, and provides the model and the basis for the research of intelligent robot. The control algorithm of real time control of MSC1210 MCU and PID, make sure tracking line robot speed and accurate steering control, and the system has good application value.

\section{REFERENCES}

[1] LI Ming-he, Wang Wei, Wang Jian, Wang Wei, WANG Dian-jun, XUE Long, "Design of Omni-directional Mobile Robot System," J. of Anhui University of Technology(Natural Science), vol 28, no.1, pp.51-54, January,2011.

[2] Hou Yi-kun, Hou Cong-Ling, Liu Yi-kun, "The Design of an Intelligent Car Control System Based on Embedded System ,' MECHANICAL \& ELECTRICAL ENGINEERING TECHNOLOGY, vol 39, no.1, pp.21-23, January,2010.

[3] YU Xiang-zhen, LI Mei-fang, Study of Neuro-Fuzzy Network Control of a Flexible Robot[J], JOURNAL OF UNIVERSITY OF ELECTRONIC SCIENCE AND TECHNOLOGY OF CHINA, 2005, 34(2)

[4] CHEN Su-hua, FANG Xu, SI Hong-chang. "Monitoring System of Intelligent Car Based on ARM and FPGA," Modern Electronics Technique, vol 33, no.16, pp.59-62, Aug, 2010.

[5] YANG Gui-lin, "Design of the Intelligent Vehicle Based on AT89S52," MICROCOMPUTER INFORMATION, vol 26, no.20, pp.124-125, Jul, 2010.

[6] Jiang Ziyun, Zhang Wei, et al. A Test Program of MSC1210 Intelligence Controller, IMP \& HIRFL Annual Repor,2012.

[7] Jiang Ziyun, Zhang Wei, et al. Optimized Program of MSC1210 Power Supply Controller. Nuclear Physics Review, vol 30, no.1, pp.38-41, March,2013.

[8] Chang, C.C., Kai-Tai Song. ULTRASONIC sensor data integration and its applocation to environment perception. Journal of Robotic Systems, vol 13, no.10, pp.663-677, 1996.

[9] S. Colantonio, M. Benvenuti, M.G. Di Bono, et. al. Object tracking in a stereo and infrared vision system. Infrared Physics \& Technology, 49(2007): 266-271

[10] Shu Huailin, On the Ability the PID Neural Networks in Nonlinear System Identification[J], GUANGDONG AUTOMATION \& INFORMATION ENGINEERING, 2001, 22(4) 